\title{
Starbursts at intermediate redshifts
}

\author{
Duilia de Mello ${ }^{1,2}$ and The GOODS Team ${ }^{3}$ \\ ${ }^{1}$ LASP NASA's GSFC Greenbelt, MD20771, USA \\ ${ }^{2}$ Catholic University of America and Johns Hopkins University, USA \\ 3 The Great Observatories Origins Deep Surveys
}

\begin{abstract}
In this work I present the survey which is searching for the rise in the volume-averaged star formation rate at intermediate redshifts $(\mathrm{z}<1)$. This survey is a combination of the data taken with Advanced Camera for Surveys in parallel with WFPC2 (U band) of the GOODS fields. We use template fitting of the spectral energy distributions to obtain photometric redshifts and classify the objects as starbursts, early- and late-types. The colors of the starbursts were reproduced and ages were estimated with the stellar evolutionary synthesis code Starburst99. By matching the optical catalog with the Chandra X-ray catalog we have identified the Xray sources and possible candidates for having a combination of active nuclei and starburst at intermediate redshifts. Analysis of the light concentration, asymmetry and clumpiness, shows that this sample is a mixed bag, containing dwarf ellipticals, early and late-spirals and peculiar objects which resemble mergers in progress. These results have strong implications for galaxy evolution since it shows for the first time who are the candidates for the rise of the SFR at intermediate-z.
\end{abstract}

\section{Why Starbursts?}

Starbursts are energetically important: within $10 \mathrm{Mpc}$ of the Galaxy $25 \%$ of the high mass star formation occurs in starburst galaxies (Heckman 1997). Moreover, they are the contributors to the rise in the cosmic star formation density between $0<\mathrm{z}<1$. This is a particularly important epoch in galaxy evolution which is often attributed to a disk epoch when gaseous galactic disks are converted into stars (Madau et al. 1996). The fate of these galaxies is unknown. They could either fade away and would not be seeing at $z=$ 0 or merge to form larger systems. Our goal is to explore the evolution of these starbursts at intermediate redshifts.

\section{GOODS}

The 'Great Observatories Origins Deep Survey' (GOODS) collects data with the largest telescopes in the world in two fields the Chandra Deep Field South (CDF-S) and the Hubble Deep Field North. In this work we will concentrate our analysis in the CDFS. The CDF-S contains the deepest region recently observed by HST, the Ultra Deep Field (UDF), and is likely to be a target for most of the large observatories generating unprecedented wealth of data. The deep GOODS images of the CDF-S which were taken during five epochs with HST Advanced Camera for Surveys (ACS) in B (F435W), V (F606W), I (F775W), and z (F850W) (Giavalisco et al. 2004) reveal 30,000 objects within 24 arcminutes square.

\subsection{Starburst at Intermediate redshifts}

A starburst sample was selected from GOODS/ACS multiwaveband when obtaining photometric redshifts (Mobasher et al. 2004a, b). The photometric redshift method chosen 


\begin{tabular}{ccc} 
Type & Concentration & Asymmetry \\
\hline Late-types (65\%) & 2.65 & 0.27 \\
Early-types (21\%) & 2.35 & 0.23 \\
Starbursts (14\%) & 2.53 & 0.33 \\
\hline U-bright & 2.63 & 0.35 \\
\hline
\end{tabular}

Table 1. Morphology Analysis. Top part refers to galaxies in Mobasher et al. 2004a. Bottom part refers to the U-bright sample

(Benitez 2000) includes templates of the spectral energy distribution of E, Sbc, Scd, Im (Coleman et al. 1908) and two starbursts (Kinney et al. 1996). From the template fitting of the SEDs, they found 157 starbursts and 977 as other types. This optically selected sample is made of relatively blue starbursts, it is complete to $\mathrm{R}_{A B} \leqslant 24$ and extends to $\mathrm{z} \sim 1$. A large fraction $(50 \%)$ of the starbursts have high asymmetry compared to early- $(13 \%)$ and late-types $(27 \%)$, suggesting that like in ultra-luminous infrared galaxies (ULIRGS) their star formation is probably tidally triggered.

\section{U-bright sample, Morphology and Age}

The lack of near-UV coverage by ACS was compensated by taking parallel WFPC2 (F300W) images whenever ACS was pointing at the GOODS fields (de Mello et al. 2004). However, due to the GOODS multi-epoch visits and the fact that WFPC2(F300W) was observing in parallel, each visit corresponded to a different area of the CDF-S with a heterogeneous coverage. We have inspected these images and compiled a U-selected sample of objects with photometric redshifts $\mathrm{z}<1$ for which we have ACS counterparts. As seen in Fig.1, the deep ACS images suggest that these galaxies have a variety of morphologies, from early-types to late-types (de Mello et al. 2004). A gallery of objects is shown in http://goods.gsfc.nasa.gov/goods/duilia/gallery/.

Galaxy morphology was evaluated by measuring the light concentration $(\mathrm{C})$, asymmetry (A) and clumpiness (S) of the ACS images in the restframe B band using the method by Conselice (2003). Early-type galaxies are expected to have a high concentration index and low asymmetries whereas late-type (disks) galaxies have low light concentration and high asymmetries. As suggested by Conselice et al. (2000), a correlation between asymmetry and color can be seen for most of Hubble types and outliers which are too asymmetric for their colors are probably dynamically disturbed.

In Table 1 we show the average values of concentration and asymmetry for all detected objects in GOODS/CDF-S at $0.24<z<1.3$. We found that galaxies in the former sample are on average more asymmetric than all starbursts in Mobasher et al. (2004a).

The stellar populations of the U-brigth galaxies were dated using the evolutionary synthesis code Starburst99 (Leitherer et al. 1999). Models of the emission from starburst stellar populations were generated as a function of age, IMF, and metallicity. Color-color plots of models and data enabled dating the stellar population of these starbursts. Models with a continuous star formation law at a constant rate (1 solar mass per year) over 10 Gyr were tested. Extinction of $\mathrm{E}(\mathrm{B}-\mathrm{V})=0.2,0.25,0.3,0.35$ and a Calzetti law was applied to the models. We found that all U-bright objects have ages $<1 \mathrm{Gyr}$. 

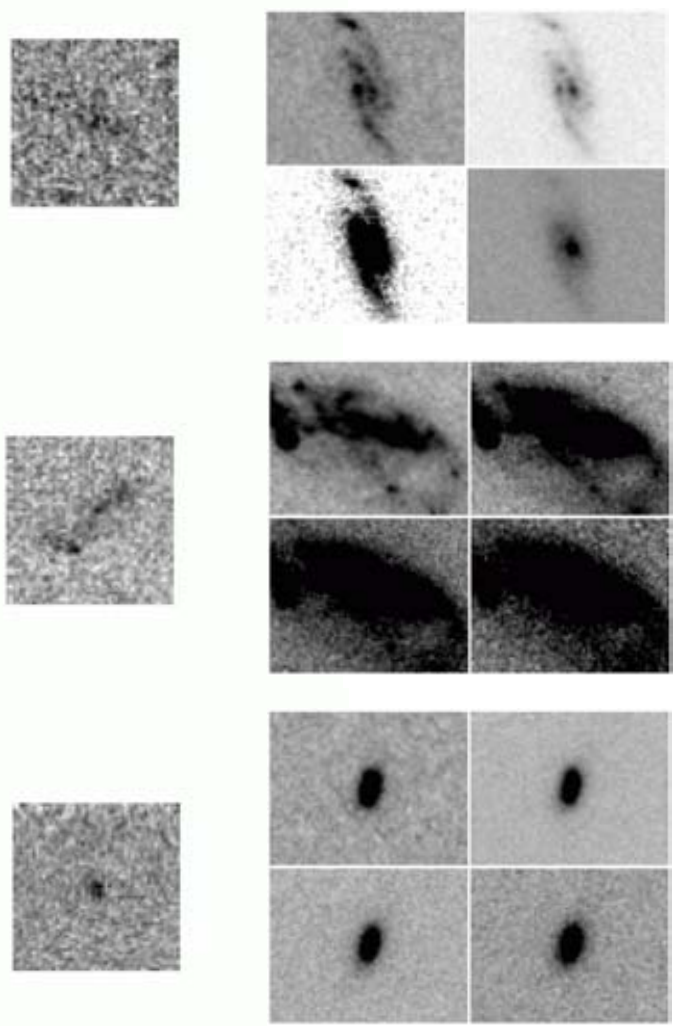

Figure 1. Examples of galaxies in the WFPC2 U parallels (left side) and their counterparts in the HST/ACS images (B, V, I, z - starting from left, clockwise).

\section{X-ray Sources}

By matching the near-UV sample with the ACS objects with X-Ray emission provided by the GOODS team (Koekemoer et al. 2004) we found three X-ray sources. We adopted 3.0 arcsec as the maximum offset radius between the U and the ACS z-band coordinates. Visual inspection (Fig. 2) of the three X-ray sources shows that one of the objects in the ACS catalog is a star-like object and the other two are late-type galaxies. The star-like object is one of the most powerful CXO sources (Giaconni et al. 2002) in the entire ACS field (Soft X-ray flux=4.00e-14 $\pm 4.69 \mathrm{e}-16$ and Hard X-ray flux 8.06e-14 $\pm 1.65 \mathrm{e}-15$ ), it is a TypeI AGN at $\mathrm{z}=0.544$.

\section{Conclusion}

We conclude that galaxies going through intense star formation at intermediate- $z$ come in a variety of morphology from early- to late-type, they have stellar population with ages $<1 \mathrm{Gyr}$, they are asymmetric and can be X-ray sources.

\section{Acknowledgements}

Thanks to the organizers of the IAU 222 and to the GOODS team. Support for this work was provided by NASA through grants GO09583.01-96A and GO09481.01-A from 

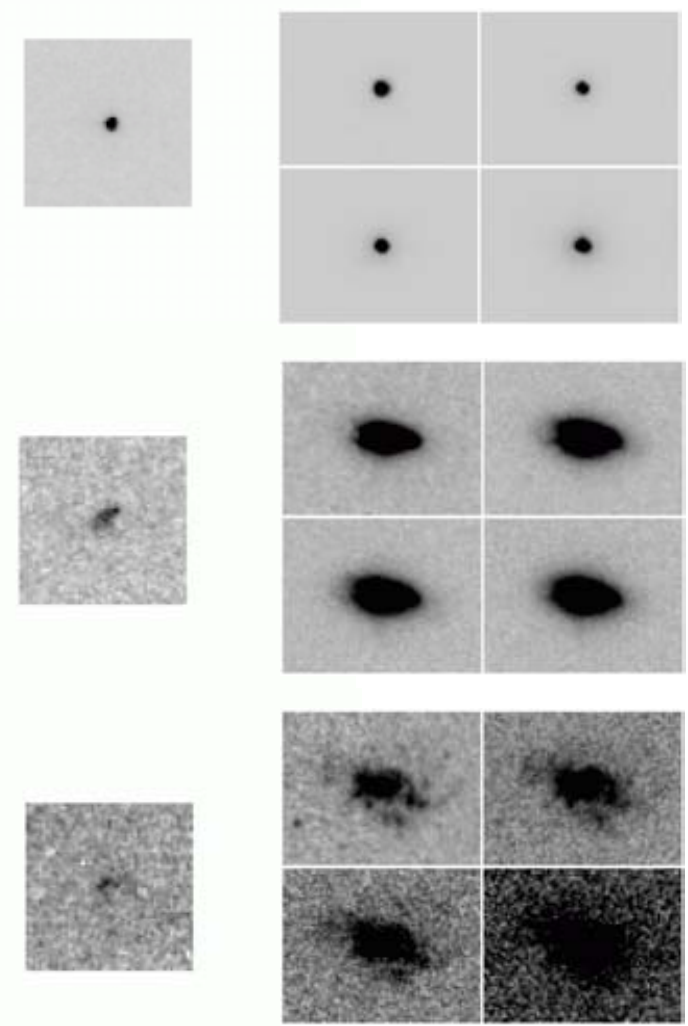

Figure 2. X-Ray sources in the WFPC2 U parallels (left side) and their counterparts in the HST/ACS images (B, V, I, z - starting from left, clockwise).

the Space Telescope Science Institute, which is operated by the Association of Universities for Research in Astronomy, under NASA contract NAS5-26555.

\section{References}

Benitez, N. 2000, ApJ, 536, 571

Coleman, G. D., Wu, C-C., \& Wedman, D. W. 1980, ApJS, 43, 393

Conselice, C. 2003, ApJS, 147, 1

Conselice, C., Bershady, M. A., \& Gallagher, J. S. III. 2000, A\&A, 354, L21

Giacconi, R., et al. 2002, ApJS, 139, 369

Giavalisco, M., et al. 2004, ApJ, 600, L93

Heckman, T. M. 1997, Starbursts and Cosmology. in Star Formation Near and Far, the 7th Annual Astrophysics Conference in Maryland, (eds. S. S. Holt and L. G. Mundy)

Kinney, A., et al. 1996, ApJ, 467, 38

Koekemoer, A. 2004, ApJ, 600, L123

Leitherer, C., et al. 1999, ApJS, 123, 3 (Starburst99)

Madau, P., et al. 1996, MNRAS, 283, 1388

Mobasher, B., et al. 2004a, ApJ, 500, L143

Mobasher, B., et al. 2004b, ApJ, 600, L167 